\title{
Optimal Flow and Pressure Management in Machine Perfusion Systems for Organ Preservation
}

\author{
Ivo C. J. H. Post, ${ }^{1}$ Marcel C. Dirkes, ${ }^{1}$ Michal Heger, ${ }^{1}$ Rick Bezemer, ${ }^{2}$ Johan van 'T Leven, ${ }^{3}$ \\ and Thomas M. van GuliK ${ }^{1}$ \\ ${ }^{1}$ Department of Surgery (Surgical Laboratory), Academic Medical Center, University of Amsterdam, Meibergdreef 9, \\ 1105 AZ Amsterdam, The Netherlands; ${ }^{2}$ Department of Translational Physiology, Academic Medical Center, University of \\ Amsterdam, Amsterdam, The Netherlands; and ${ }^{3}$ Cori-Tech, Bronkhorst High-Tech BV, Ruurlo, The Netherlands
}

(Received 26 February 2012; accepted 19 May 2012; published online 6 June 2012)

Associate Editor Dan Elson oversaw the review of this article.

\begin{abstract}
Intra-organ flow is the most critical parameter in machine-perfused organ preservation systems (MPS). Ultrasonic flow sensors (UFS) are commonly employed in MPS. However, UFS are sensitive to changes in fluid composition and temperature and require recalibration. Novel Coriolistype mass flow sensors (CFS) may be more suitable for MPS because the measurement technique is not amenable to these factors. The effect of viscosity, colloids, temperature, pressure, and preservation solution on flow measurement accuracy of UFS and CFS was therefore investigated. A CFSbased MPS was built and validated for setpoint stability using porcine kidneys and the ability to reproduce different pressure and flow waveforms. The UFS exhibited a temperature- and preservation solution-dependent overestimation of flow rate compared to the CFS. The CFS deviated minimally from the actual flow rate and did not require recalibration. The CFSbased MPS conformed to the preprogrammed temperature, flow, pressure, and vascular resistance settings during 6-h kidney preservation. The system was also able to accurately reproduce different pressure and flow waveforms. Conclusively, CFS-based MPS are more suitable for organ preservation than UFS-based MPS. Our CFS-based MPS provides a versatile yet robust experimental platform for testing and validating different types of clinical and experimental MPS.
\end{abstract}

Keywords-Normothermic, Hypothermic, Coriolis flow sensor, Ultrasonic flow sensor, Waveform.

\section{ABBREVIATIONS}

NHBD Non-heartbeating donor

HMP Hypothermic machine perfusion $\left(4^{\circ} \mathrm{C}\right)$

Address correspondence to Thomas M. van Gulik, Department of Surgery (Surgical Laboratory), Academic Medical Center, University of Amsterdam, Meibergdreef 9, 1105 AZ Amsterdam, The Netherlands. Electronic mail: T.M.vanGulik@amc.uva.nl

$\begin{array}{ll}\text { SCS } & \text { Static cold storage }\left(4^{\circ} \mathrm{C}\right) \\ \text { NMP } & \text { Normothermic machine perfusion }\left(37^{\circ} \mathrm{C}\right) \\ \text { VR } & \text { Vascular resistance }\left(\mathrm{mmHg} \mathrm{mL} \mathrm{min}^{-1} \mathrm{~min}^{-1}\right) \\ \text { DW } & \text { Demineralized water } \\ \text { HTK } & \text { Histidine-tryptophan-ketoglutarate } \\ \text { PS } & \text { Polysol } \\ \text { UW } & \text { University of Wisconsin solution } \\ \text { RL } & \text { Ringer lactate } \\ F_{\mathrm{c}} & \text { Coriolis force }\left(\mathrm{N} \mathrm{m}^{-1} \mathrm{~s}^{-1}\right) \\ L & \text { Length (m) } \\ \Omega & \text { Greek, small letter Omega, torsion (degrees } \\ & \text { angulation) } \\ \Phi_{\mathrm{m}} & \text { Greek, capital letter Phi, mass flow } \\ & \left(\mathrm{kg} \mathrm{h}{ }^{-1}\right)\end{array}$

\section{INTRODUCTION}

A chronic shortage of donor organs has been the driving force for an expansion of the donor pool with non-heartbeating donor (NHBD) organs. ${ }^{13}$ Due to their origin, these organs are subjected to extensive organ damage as opposed to organs retrieved from heartbeating or living donors. Despite the advantages of hypothermic $\left(4{ }^{\circ} \mathrm{C}\right)$ organ preservation, the clinical standard in transplantation programs, hypothermia leads to postpreservation organ damage following transplantation and reperfusion due to intracellular energy-, metabolite-, and anti-oxidant shortage. ${ }^{3,22}$ As a consequence, renewed interest in ex vivo rewarming prior to transplantation and (sub)normothermic machine perfusion $\left(32-37^{\circ} \mathrm{C}\right)$ has arisen with the aim to prevent the detrimental effects associated with hypothermia. $5,12,19-21$

For use under (sub)normothermic conditions, preservation solutions must contain metabolismsupporting substrates, colloids to prevent edema, and 
ideally, oxygen carriers. ${ }^{8}$ The complexity of these solutions has implications for the dynamics of machine perfusion (i.e., pressure, flow, and waveform) that in turn, may affect accuracy of flow measurements in preservation solutions due to their temperaturedependent rheological properties. ${ }^{2,18}$

Perfusate flow remains the most commonly used parameter for the quality of organ perfusion. Accuracy of flow measurement, therefore, is imperative since flow and pressure are used to calculate intra-organ vascular resistance (VR, $\mathrm{mmHg} \mathrm{mL}{ }^{-1} \mathrm{~min}^{-1}$ ) that is regarded the main indicator of micro-vascular integrity. ${ }^{4,6,9,17}$

Ultrasonic-based sensors are usually applied in the experimental setting of hypo- or normothermic machine perfusion. Although they are easy-to-use and comprise a broad array of clamp-on tubing sensors, changes in perfusion temperature, pressure, and applied preservation solution can affect the accuracy of flow measurements. ${ }^{8} 10$ In addition, ultrasonic sensors require recalibration for temperature and solution changes, restricting their application in organ resuscitation by ex vivo rewarming prior to implantation. Using a Coriolis-based mass flow sensor that measures flow based on the solutions' mass instead of volume or the Doppler effect may circumvent these drawbacks. Due to this property, experimental machine perfusion systems with Coriolis-based flow sensors might enable reproduction of clinically and experimentally encountered flow dynamics with a greater accuracy under changing preservation conditions. ${ }^{1}$

The aim of this study was to determine the accuracy of flow measurements using ultrasonic- and Coriolisbased flow sensors with respect to temperaturedependent viscosities and pressure variations of the following preservations solutions: University of Wisconsin (UW), histidine-tryptophan-ketoglutarate (HTK), and the experimental preservation solution, Polysol (PS). On the basis of these findings, an experimental machine perfusion system incorporating Coriolis-based mass flow technology was developed and validated for temperature, flow, and pressure stability with the final goal to provide reproducible generation of versatile flow patterns (waveforms). This development intended to provide a platform for optimal perfusion parameter determination.

\section{MATERIALS AND METHODS}

\section{Viscosity Measurements in Preservation Solutions}

Temperature and preservation solution-dependent viscosity was measured using a LV DV-II + Pro Digital Rheometer (Brookfield Engineering Laboratories, Middleboro, MA) and Rheocalc 3.1-1 (Brookfield Engineering Laboratories) software for data acquisition.
Samples of preservation solution $(500 \mu \mathrm{L})$ were transferred to the measurement cup that was kept at 4, 15, 20, 28 , or $37^{\circ} \mathrm{C}$ using a water bath (Tamson Instruments, Zoetermeer, The Netherlands). Temperaturedependent viscosity ( $N=7$ for each solution) was determined in demineralized water (DW, Baxter, Deerfield, IL), histidine-tryptophan-ketoglutarate (HTK, Dr F Köhller-Chemie, Bensheim, Germany), Polysol (PS, Mediphenix, Amsterdam, The Netherlands), and University of Wisconsin solution (UW, Fresenius Hemocare, Emmer-Copascuum, The Netherlands). The characteristics of the solutions are provided in Table 1.

\section{Assessment of Flow Measurement Accuracy}

Accuracy of flow measurement was determined in a custom-built setup in which two commercial systems of equal measurement capacity $\left(250 \mathrm{~mL} \mathrm{~min}^{-1}\right)$ were placed according to the manufacturers' instructions, being an ultrasonic in-line sensor (ME4PXN, Transonic, Ithaca, NY on a TS410 console) and a Coriolis mass flow controller (Cori-Flow M14; Bronkhorst Cori-Tech BV, Ruurlo, the Netherlands). Flow was generated using a non-pulsatile gearpump (DGS.11; Tuthill, Alsip, IL) and allowed to stabilize at approximately $50 \mathrm{~mL} \mathrm{~min}{ }^{-1}$. Pressure was controlled by a pressure transducer (El-Press P-502C-350R; Bronkhorst High-Tech, Ruurlo, the Netherlands) at 30, 55, 75 , or $95 \mathrm{mmHg}$ (zero stability has been determined by the manufacturer) and temperature was set to 4, 15, 20, or $28{ }^{\circ} \mathrm{C}$ using a water bath (Tamson Instruments). Flow measurement accuracy was related to the flow rate derived from a precision weighing scale (PR8002; Mettler-Toledo, Tiel, the Netherlands) that was continuously read-out by a custom-built program (Bronkhorst High-Tech, Ruurlo, the Netherlands). The program calculated the volumetric flow $\left(\mathrm{mL} \mathrm{min}{ }^{-1}\right)$ reference by means of the weight increase and density (measured prior to each experiment) of each solution.

The ultrasonic sensor was recalibrated according to the manufacturers' instructions prior to each change of solution and temperature, whereas the Coriolis sensor (manufactured and calibrated in 2009) did not require any recalibration according to its design. All hardware components were controlled by a FlowBus module (Bronkhorst High-Tech) and data were collected in FlowPlot (version 3.28, Bronkhorst).

\section{Experimental Machine Perfusion System}

A schematic overview of our custom-engineered machine perfusion system is depicted in Fig. 1. Briefly, $1 \mathrm{~L}$ of perfusion solution was kept in a glass reservoir, oxygenated by a custom-built glass oxygenator (passive diffusion-based), and two custom-built glass 
TABLE 1. Composition of solutions used.

\begin{tabular}{|c|c|c|c|c|c|}
\hline & HTK & UW & PS & $R L$ & DW \\
\hline \multicolumn{6}{|l|}{ Colloids $\left(\mathrm{g} \mathrm{L}^{-1}\right)$} \\
\hline PEG & & & 20 (35 kDa) & & \\
\hline HES & & 50 & & & \\
\hline \multicolumn{6}{|l|}{ Impermeants (mmol L $\left.{ }^{-1}\right)$} \\
\hline Mannitol & 38 & & & & \\
\hline Lactobionate & & 100 & & & \\
\hline Raffinose & & 30 & 3.2 & & \\
\hline Trehalose & & & 5.3 & & \\
\hline Sodium gluconate & & & 75 & & \\
\hline Potassium gluconate & & & 20 & & \\
\hline \multicolumn{6}{|l|}{ Buffers $\left(\mathrm{mmol} \mathrm{L}^{-1}\right)$} \\
\hline Histidine & 198 & & 6.3 & & \\
\hline $\mathrm{KH}_{2} \mathrm{PO}_{4}$ & & 25 & & & \\
\hline $\mathrm{NaH}_{2} \mathrm{PO}_{4}$ & & & 21.7 & & \\
\hline HEPES & & & 24 & & \\
\hline \multicolumn{6}{|l|}{ Electrolytes $\left(\mathrm{mmol} \mathrm{L}^{-1}\right)$} \\
\hline Calcium & 0.0015 & & & 1.5 & $<2$ \\
\hline Chloride & 32 & 20 & & 109 & $<2$ \\
\hline Magnesium & 4 & & & & $<2$ \\
\hline Magnesium sulfate & & 5 & & & \\
\hline Potassium & 9 & 120 & 15 & 4 & $<2$ \\
\hline Sodium & 15 & 25 & 120 & 130 & $<2$ \\
\hline \multicolumn{6}{|c|}{ Anti-oxidants (mmol L $\left.{ }^{-1}\right)$} \\
\hline Tryptophan & 2 & & & & \\
\hline Allopurinol & & 1 & & & \\
\hline Glutathione & & 3 & 5.6 & & \\
\hline Ascorbic acid & & & 0.11 & & \\
\hline Alpha-tocopherol & & & $5.4 \times 10^{-5}$ & & \\
\hline \multicolumn{6}{|l|}{ Additives $\left(\mathrm{mmol} \mathrm{L}^{-1}\right)$} \\
\hline Ketoglutarate & 1 & & & & \\
\hline Adenosine & & 5 & 5 & & \\
\hline Vitamins & & & $\$$ & & \\
\hline Amino acids & & & $\$ \$$ & & \\
\hline Lactate & & & & 28 & \\
\hline Osmolarity (mOsm L ${ }^{-1}$ ) & 310 & 325 & 325 & 272 & $<1$ \\
\hline $\mathrm{pH}$ & $7.02-7.2$ & 7.4 & 7.4 & 6.5 & 7 \\
\hline
\end{tabular}

HTK: Histidine-tryptophan-ketoglutarate; UW: University of Wisconsin solution; PS: Polysol; RL: Ringers lactate; DW: Demineralized water; PEG: Polyethylene glycol; HES: Hydroxyethyl starch.

$\$\left(\mathrm{mmol} \mathrm{L}^{-1}\right)$ : ascorbic acid (0.11), biotin (0.21), Ca-pantothenate (0.004), choline chloride $(0.01)$, inositol $(0.07)$, ergocalciferol $\left(3 \times 10^{-4}\right)$, folic acid $(0.002)$, menadione $\left(4 \times 10^{-5}\right)$, nicotinamide (0.01), nicotinic acid (0.004), pyridoxal (0.005), riboflavin $(0.003)$, thiamine (0.03), vitamin A $\left(3 \times 10^{-4}\right)$, vitamin B12 $\left(1 \times 10^{-4}\right)$, and vitamin $\mathrm{E}\left(5 \times 10^{-5}\right)$.

$\$ \$\left(\mathrm{mmol} \mathrm{L}^{-1}\right)$ : alanine (1.01), arginine (1.18), asparagine (0.08), aspartic acid (0.23), cysteine (0.33), glutamic acid (0.34), leucine $(0.57)$, glutamine $(0.002)$, glycine $(0.67)$, isoleucine $(0.38)$, lysine $(0.48)$, methionine $(0.30)$, ornithine $(2.00)$, phenylalanine $(0.30)$, proline $(0.78)$, serine $(0.29)$, threonine $(0.34)$, tryptophan $(0.88)$, tyrosine (0.19), and valine (0.43)

bubble traps. Perfusate temperature was controlled by a custom-built glass heat exchanger. The perfusion system was driven by a rollerpump (Watson-Marlow, Wilmington, MA), pumping perfusion solution from the reservoir to the first bubble trap while a miniature membrane pump (NF60KPDCB-4, KNF Neuberger GMBH, Freiburg, Germany) provided the arterial perfusion pressure after the first bubble trap. The membrane pump was combined with two bypasses in order to provide non-pulsatile flow to the organ.

All glass components were temperature-controlled by a waterbath (HMT-200; HETO, Holten, the Netherlands) and thermocouple (C8.B Licox; Integra, Vilvoorde, Belgium). A pressure controller (El-Press P-502C-350R; Bronkhorst) was positioned directly prior to the glass organ chamber for pressure regulation. A gas flow controller $\left(\mathrm{O}_{2}\right.$ El-Press F-201CV; Bronkhorst) coupled to a 50-L oxygen-containing cylinder (Hoekloos Medical, Amsterdam, the Netherlands) subsequently regulated oxygenation of the perfusate. All hardware components were connected to a data acquisition board (NI USB-6229 BNC, National Instruments, Austin, TX) and controlled by a custom-written program in Labview (version 8.4.2, National Instruments).

\section{Stability of the Machine Perfusion System Settings}

System setting stability was determined using a nonpulsatile flow of $50 \mathrm{~mL} \mathrm{~min}{ }^{-1}$ in a pressure range of 0 to $95 \mathrm{mmHg}$ and temperatures of $4,15,20,28$, or $37^{\circ} \mathrm{C}$. As perfusion solution, PS was used inasmuch as it was specifically designed as a colloidal machine perfusion solution. Stability was defined as the capability of the perfusion system to comply with the preprogrammed setpoint with respect to flow, pressure, and temperature. All measurements were performed over a period of $5 \mathrm{~min}$ and repeated five times in a random fashion within the same temperature group.

\section{Stability During Ex Vivo Kidney Preservation}

After approval of the animal ethics committee (BEX 102084) and in accordance with the principles of laboratory animal care, two non-heartbeating kidneys from two healthy, 50-kg female Landrace pigs were retrieved as previously described ${ }^{14}$ and connected to the preservation system. The kidneys were perfused for $6 \mathrm{~h}$ via the renal artery with free venous outflow. PS with $10 \% \mathrm{v} / \mathrm{v}$ PFOB emulsion ${ }^{11}$ as oxygen carrier was used as preservation solution at $28^{\circ} \mathrm{C}$ and a fixed perfusion pressure of $75 \mathrm{mmHg}$. Temperature, pressure, and flow were continuously measured using the previously mentioned setup.

\section{Multi-Functionality of the Experimental Machine Perfusion System}

To confirm multi-functionality of the perfusion system, complex waveforms encompassing pressure spikes or smooth pressure control were selected to ascertain the ability to mimic commercial devices with different perfusion profiles. Although rapid pressure spikes are not clinically demanded, they provide 


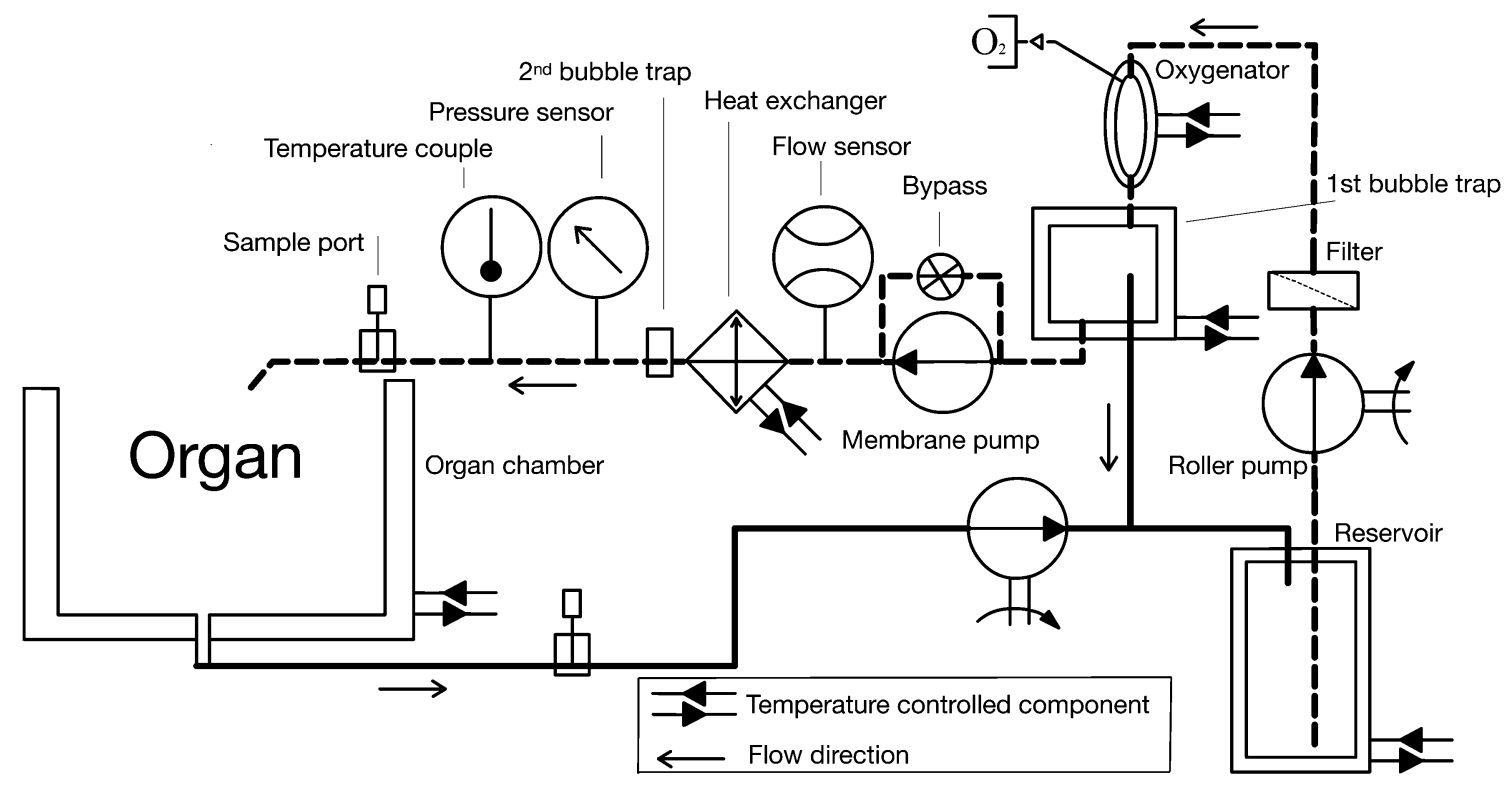

FIGURE 1. Schematic representation of the machine perfusion system. The perfusion solution in the reservoir passes a roller pump, filter, oxygenator, bubble trap, miniature membrane pump, mass flow transducer, heat exchanger, 2nd bubble trap, pressure sensor, and temperature couple prior to entering the organ's artery.

insight in the capabilities of rapid adaptations by the perfusion setup. To this end, sinusoidal, sawtooth, and block waveforms were preprogrammed in Labview and sent as setpoints to the perfusion system. The waveforms' characteristics were composed of a $10-\mathrm{mmHg}$ offset and $10-\mathrm{mmHg}$ amplitude as frequently used in the clinical setting, and the baseline flow was set at $10 \mathrm{~mL} \min ^{-1}$ to allow pressure release at a $0-\mathrm{mmHg}$ setpoint.

While most commercial perfusion preservation devices generate a sinusoidal waveform, a theoretical, more complex waveform composed of fast and smooth pressure changes combined with a stabilization phase was created to mimic pre-clinical devices like the Airdrive. ${ }^{15}$ The waveform characteristics were defined as a $15-\mathrm{mmHg}$ offset and $10-\mathrm{mmHg}$ amplitude in a onesecond timespan but with a baseline flow of $20 \mathrm{~mL} \mathrm{~min}^{-1}$ to allow pressure drops. Each waveform measurement was reproduced in tenfold in random order at $20^{\circ} \mathrm{C}$ to determine reproducibility of waveform generation by the system.

\section{Statistical Analysis}

Differences were statistically analyzed for significance using GraphPad Prism (GraphPad software, La Jolla, CA). For intra- and intergroup differences in the viscosity experiments, a Kruskal-Wallis test with post hoc Dunn's test was employed. All other ordinal differences were analyzed by a two-way ANOVA with Bonferroni post hoc test. Flow measurement accuracy was displayed using a Bland-Altman plot using the comparison: difference $(A-B)$ vs. average in which the difference is defined as the precision weighing scale result minus the Coriolis or ultrasound result, plotted against their averaged result. A $p$-value of $<0.05$ was considered statistically significant.

\section{RESULTS}

\section{Viscosity of Preservation Solutions}

Colloidal solutions showed a significant temperature-dependent viscosity while non-colloidal solutions did not (Fig. 2). The viscosity of the colloidal solutions UW and PS was 2.5 -fold higher at $4{ }^{\circ} \mathrm{C}$ compared to $37{ }^{\circ} \mathrm{C}(P<0.0001)$. This effect was less pronounced for the non-colloidal solution HTK that exhibited a 1.6 -fold difference $(P<0.0001)$. A significant difference between the viscosities of the solutions was found at all temperatures except for the non-colloidal solutions at 28 and $37^{\circ} \mathrm{C}$.

\section{Flow Measurement Accuracy}

Flow measurement accuracy of the ultrasonic- and Coriolis-based sensors related to the precision weighing scale reference was mainly determined by the solution used, as evidenced by the Bland-Altman analysis (Fig. 3).

The Coriolis mass flow sensor showed very little-tonone deviation from the flow measurement reference, 
regardless of preservation solution type and temperature. The maximum flow rate overestimation by the Coriolis sensor was $0.5 \mathrm{~mL} \mathrm{~min}{ }^{-1}$ in the $4{ }^{\circ} \mathrm{C}$ HTK group.

The ultrasonic sensor displayed a more pronounced overestimation of the flow rate at 4 and $15^{\circ} \mathrm{C}$, with a minimum of $3.7 \mathrm{~mL} \mathrm{~min}{ }^{-1}$ for $\mathrm{RL}$ and a maximum of

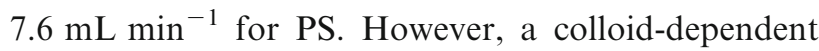

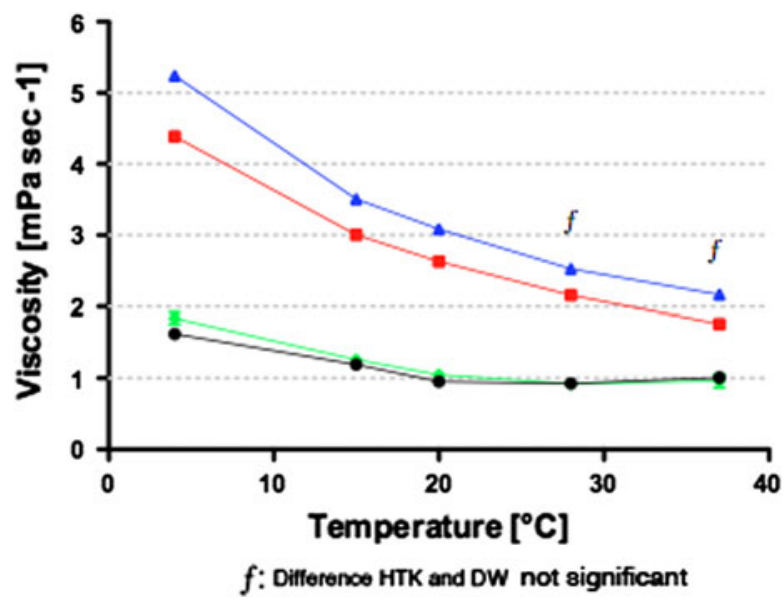

FIGURE 2. Viscosity of UW (blue), PS (red), HTK (green), and RL (black) showing a clear temperature-dependent effect. effect could not be demonstrated at these temperatures as shown by the inter- and intra-group analyses (Table 2). At higher temperatures $\left(20\right.$ and $\left.28{ }^{\circ} \mathrm{C}\right)$ the ultrasonic flow sensor (UFS) displayed greater accuracy with non-colloidal solutions (HTK and RL) in comparison to colloidal solutions (UW and PS, Table 2).

Finally, the flow rate measurement accuracy of the ultrasonic and Coriolis flow sensor was not influenced by pressure variations, regardless of temperature and the preservation solution applied (data not shown).

\section{Stability of the Experimental Machine Perfusion System Settings}

The perfusion system performed in excellent compliance with the pre-programmed setpoint (Table 3). With respect to flow stability, the measured standard deviation (SD) from the setpoint did not exceed $0.317 \mathrm{~mL} \mathrm{~min}^{-1}$ across the assessed temperature range of $4-37^{\circ} \mathrm{C}$. Regarding the perfusion pressure, the system could properly manage the pressure setpoint, indicated by the maximum pressure $\mathrm{SD}$ of $0.022 \mathrm{mmHg}$. This minimal SD from the pressure setpoint indicates the ability to provide non-pulsatile flow. Furthermore, at the $50-\mathrm{mL} \mathrm{min}^{-1}$ flow rate, the preservation solution temperature showed a maximum SD from the
$\mathrm{RL}$
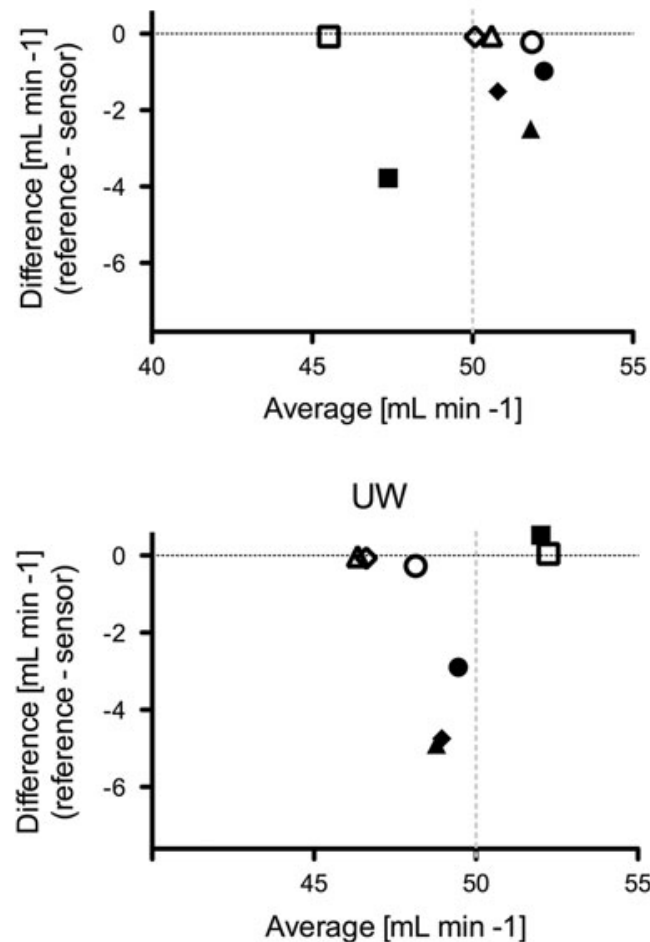

PS

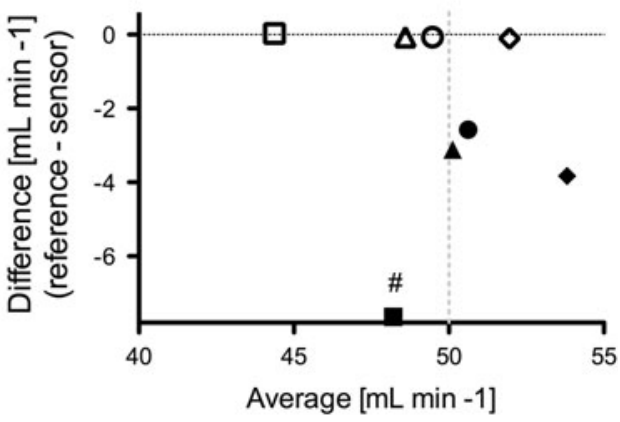

HTK
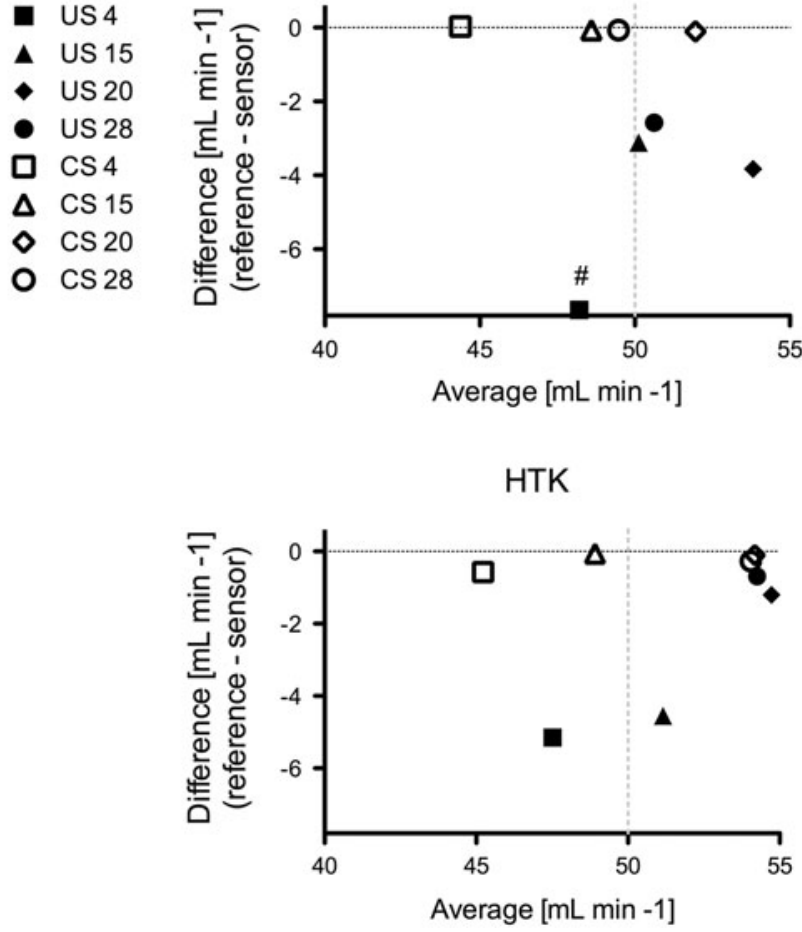

FIGURE 3. Bland-Altman plots [weighing scale-ultrasonic (US) or Coriolis (CS) vs. average] displaying overestimation of flow by the US technique in comparison to the CS technique. 
TABLE 2. Statistical differences in measurement accuracy between solutions per temperature group.

\begin{tabular}{llr}
\hline Temperature $\left({ }^{\circ} \mathrm{C}\right)$ & Solutions & $p$ value \\
\hline $\begin{array}{l}\text { Coriolis sensor } \\
20\end{array}$ & & \\
Ultrasonic sensor & PS vs. UW & 0.014 \\
4 & & \\
& PS vs. UW & $<0.001$ \\
& PS vs. HTK & $<0.001$ \\
15 & PS vs. RL & $<0.001$ \\
& PS vs. UW & $<0.001$ \\
& PS vs. HTK & $<0.001$ \\
& PS vs. RL & 0.034 \\
& UW vs. HTK & $<0.001$ \\
20 & HTK vs. RL & $<0.001$ \\
& PS vs. HTK & $<0.001$ \\
& PS vs. RL & 0.001 \\
& UW vs. HTK & $<0.001$ \\
28 & UW vs. RL & $<0.001$ \\
& PS vs. HTK & $<0.001$ \\
& PS vs. RL & $<0.001$ \\
& UW vs. HTK & $<0.001$ \\
& UW vs. RL & $<0.001$ \\
\hline
\end{tabular}

HTK: Histidine-tryptophan-ketoglutarate; UW: University of Wisconsin solution; PS: Polysol; RL: Ringers lactate; DW: Demineralized water.

TABLE 3. Machine perfusion system settings stability.

\begin{tabular}{lccc}
\hline Temperature $\left({ }^{\circ} \mathrm{C}\right)$ & Setpoint & Mean & SD \\
\hline Flow $\left(\mathrm{mL} \mathrm{min}^{-1}\right)$ & & & \\
4 & 50 & 49.950 & 0.098 \\
15 & 50 & 50.062 & 0.257 \\
20 & 50 & 49.891 & 0.170 \\
28 & 50 & 49.881 & 0.125 \\
37 & 50 & 50.036 & 0.317 \\
Pressure $(\mathrm{mmHg})$ & & & \\
& 30 & 30.005 & 0.022 \\
& 55 & 55.000 & 0.002 \\
& 75 & 75.001 & 0.003 \\
Temperature $\left({ }^{\circ} \mathrm{C}\right)$ & 95 & 94.999 & 0.003 \\
& & & \\
& 4 & 3.990 & 0.036 \\
& 15 & 14.819 & 0.411 \\
& 20 & 20.224 & 0.426 \\
& 28 & 28.040 & 0.120 \\
& 37 & 37.013 & 0.371 \\
\hline
\end{tabular}

SD: standard deviation.

setpoint of $0.426{ }^{\circ} \mathrm{C}$ at $20^{\circ} \mathrm{C}$ and even lower at the other temperatures.

Ex vivo perfusion of NHBD kidneys resulted in a stable preservation solution flow of $116 \pm 9.06$ $\mathrm{mL} \min ^{-1} 100 \mathrm{~g}^{-1}$ (mean $\pm \mathrm{SD}$ ) over the 6-h preservation period. In this time, the temperature was maintained at $28.25 \pm 0.09{ }^{\circ} \mathrm{C}$ and perfusion pressure at $74.92 \pm 0.33 \mathrm{mmHg}$. The results are displayed in Fig. 4.
Multifunctionality of the Machine Perfusion System

Sinusoidal and more complex waveforms were generated in the machine perfusion system to ascertain the ability to reproduce clinical and experimental machine perfusion devices. In Fig. 5, a clear correlation is illustrated between the preprogrammed and measured waveform pressures and associated flow rates. The $R^{2}$ value for the sinusoidal, sawtooth, block, and composed waveforms were 0.987, 0.821, 0.829, and 0.997 , respectively. The goodness of fit statistics confirms the system's capability to preprogram and execute predefined waveforms, suggesting that, when properly defined, waveforms encountered in the clinical setting can be duplicated.

In this hardware setup, however, rapid pressure changes demand a slight time delay for pressure buildup and regulation by the pump and pressure controller. Without the clinical need for such rapid pressure changes, no hardware changes were applied in the setup. In support of the latter, a setpoint delay was not found in the corresponding flow waveforms and their $R^{2}$ values $(0.983,0.893,0.954$, and 0.998 for a sinus, sawtooth, block, and composed waveform, respectively). Therefore, perfusion was properly maintained despite a lag-phase in pressure control.

\section{DISCUSSION}

This study established that an inverse proportional relationship exists between preservation solution viscosity and temperature, an effect most pronounced in the colloid-containing solutions. Furthermore, temperature and type of preservation solution considerably affected flow measurement accuracy when using an ultrasonic-based flow sensor but not a Coriolisbased mass flow sensor. Incorporation of the Coriolisbased sensor in our experimental machine perfusion system proved to perform in compliance with preprogrammed flow, pressure, and temperature. Moreover, compliance with complex waveforms enabled the system to recreate well-defined flow patterns of commercially available and experimental machine preservation systems.

In experimental perfusion preservation systems the ultrasonic-based flow sensors are frequently applied and pose no risk for impaired measurement accuracy when the preservation solution and applied temperature are left unchanged. However, with the hypothermia-related detrimental effects on preserved organs during reperfusion, interest in the complex process of organ rewarming prior to reperfusion is increasing. For the application of (sub)normothermic preservation, the effects of oxygen-carrying particles, changing 
(a)

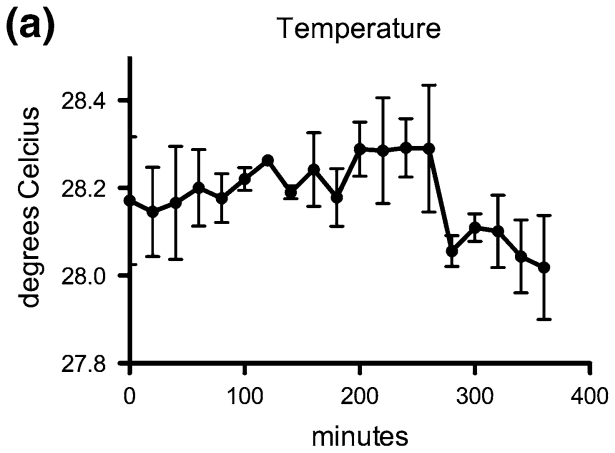

(c)

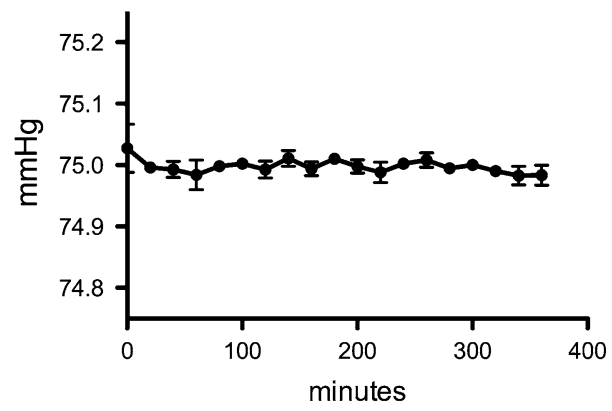

(b)

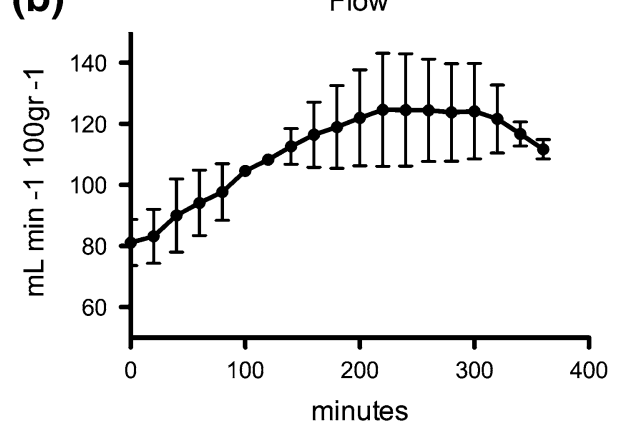

(d)

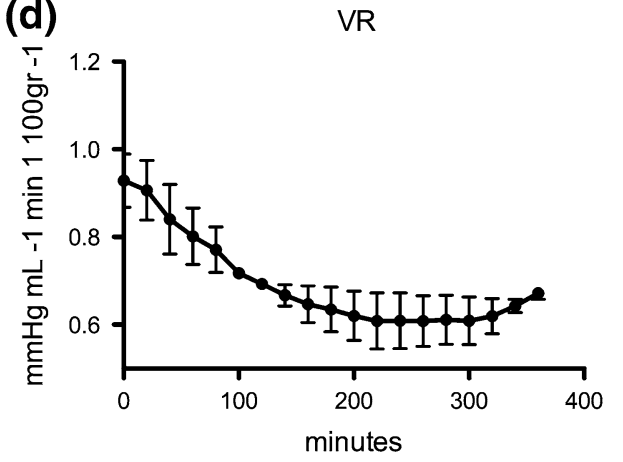

FIGURE 4. The dynamics of temperature (a), flow (b), pressure (c), and vascular resistance (VR, d) during $6 \mathrm{~h}$ ex vivo machine perfusion preservation of $2 \mathrm{NHBD}$ porcine kidneys at $28^{\circ} \mathrm{C}$.

perfusion solutions, and perfusion temperatures must be taken into account. ${ }^{7}$ In this respect, changes in the solution's composition impact the density and viscosity, leading to a change in conductivity of the ultrasonic wave used in ultrasonic-based sensors. ${ }^{2,18}$ Changes in viscosity, in turn, can cause velocity variations across the tubing diameter due to friction at the tubing walls, impairing the flow rate measurement accuracy in older generation sensors. ${ }^{10,23}$ More importantly, particles present in preservation solutions (microbubbles, protein aggregates, residual erythrocytes, artificial oxygen carriers, etc.) are constantly influencing the ultrasonic flow measurement during perfusion, which impacts its reliability. ${ }^{2}$

The major advantage of Coriolis-based flow sensors over ultrasonic sensors is that their measurement is based upon mass flow instead of volume or velocity (Doppler). Coriolis-based sensors are therefore capable to correct for temperature, density, and particle-related changes of the solutions flowing through the sensor. In a Coriolis flow sensor, two U-shaped tubes are counter-vibrating within an $80-$ to $1000-\mathrm{Hz}$ range, depending on the flow tube size. During flow, the vibration-amplitude at the inflow of the flow tube becomes 'out-of-sync' with the outflow amplitude due to the Coriolis force that moves the tube in a direction perpendicular to the flow and imposed rotational axis (Fig. 6). The Coriolis effect thereby facilitates the calculation of the exact mass flow from the phase-shift that occurs using the formula $F_{\mathrm{c}}=-2 L \omega \Phi_{\mathrm{m}}$ in which, $L$ is the flow tube length, $\omega$ the torsion applied, and $\Phi_{\mathrm{m}}$ the resulting mass flow (Fig. 7). Mass flow can subsequently be converted to volumetric flow by dividing it by the density of the fluid. Density is easily extrapolated because the given vibration frequency is dependent on the given mass of the flow tubes and the unknown density of the solution passing through the flow tubes.

The abovementioned corrections performed in a Coriolis-based flow sensor make the required recalibration prior to solution- or temperature-changes, which are obligatory for ultrasonic sensors, redundant. This is advantageous when normothermic organ resuscitation is applied, especially when taken into account that rewarming can take up to 68 min. ${ }^{16}$

The reproduction of various pressure and flow waveforms using the Coriolis technique with automatic, auto-feedback pressure regulation enabled the simulation of other experimental and commercial perfusion preservation devices. This allows research into optimal preservation conditions in one apparatus with inclusion of the current, clinically employed machine preservation devices. However, the application of Coriolis-based sensors for clinical organ preservation is currently not possible for technical reasons. The current layout of the sensor and especially the flow tube comprises frequent bends and dead volumes. With the strict clinical regulations and the inability to 
(a)

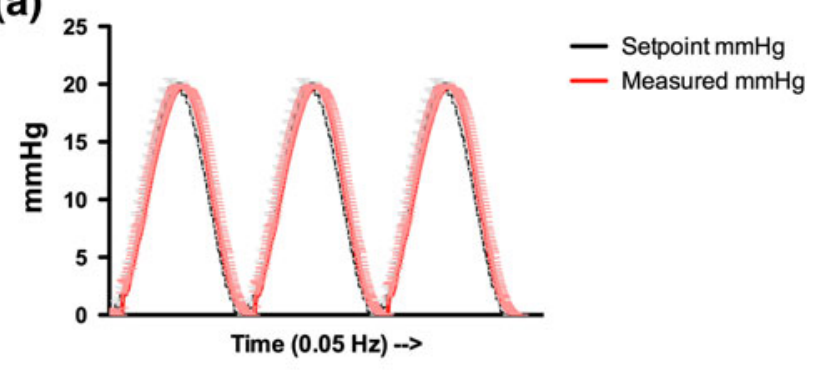

(b)

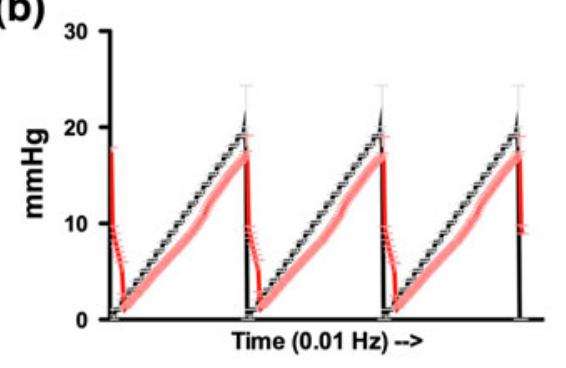

(c)

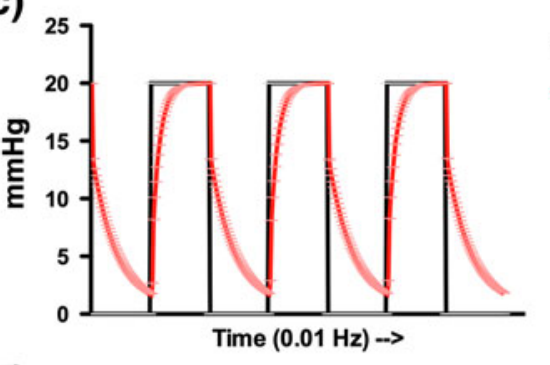

(d)
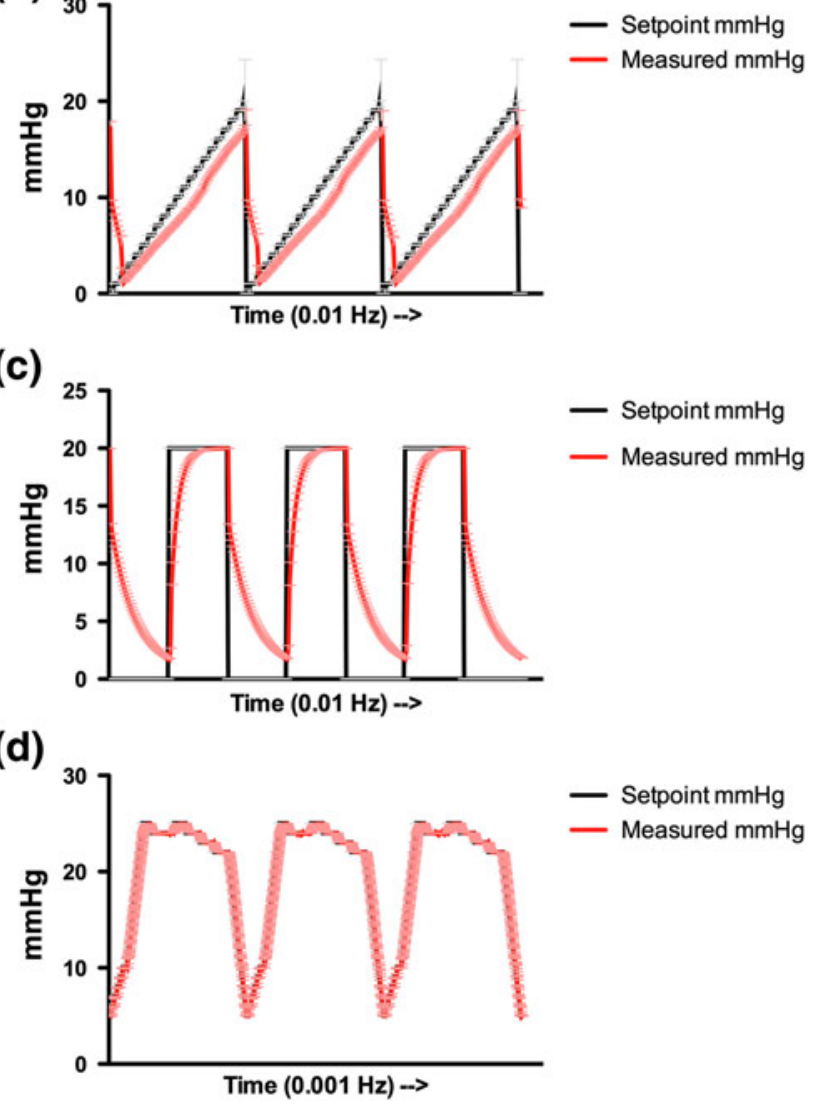

- Setpoint mmHg
— Measured mmHg (e)

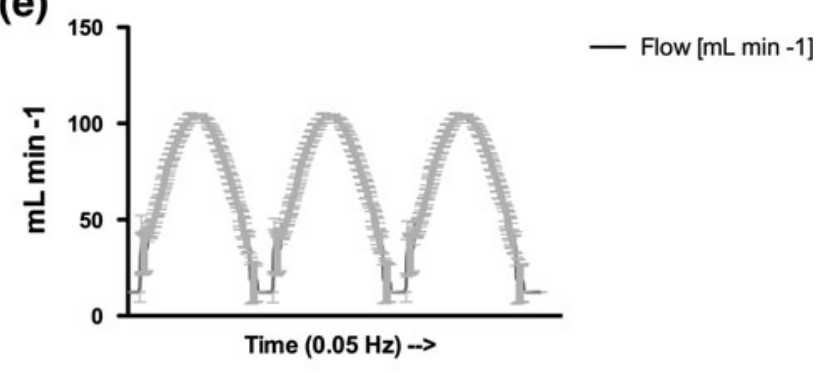

(f)

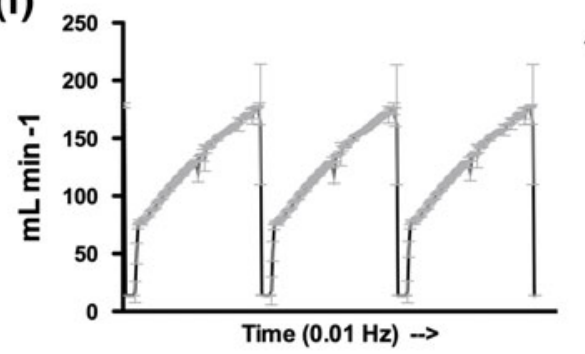

(g)

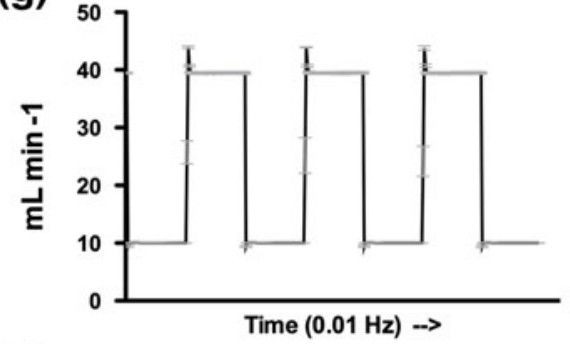

(h)

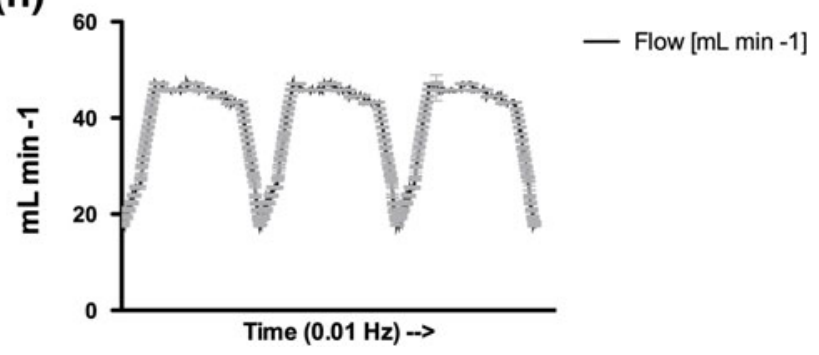

FIGURE 5. Waveform generation in the experimental system simulating a sinusoidal (a), sawtooth (b), block waveform (c), and complex composed waveform (d). The red line represents measured pressure $(\mathrm{mmHg})$ and the black line represents the setpoint given to the controller device. (e)-(h) show the associated flow patterns $\left(\mathrm{mL} \mathrm{min}^{-1}\right)$.

autoclave the sensor with the attached electronics, clinical sterility guidelines cannot be followed. Furthermore, as the sensor's measurement principle is based on the distortion of a generated vibration, the coriolis-based measurement is sensitive to external vibrations. Without hardware changes to overcome these issues, implementation in a portable, clinically useable system is not feasible. Therefore, this flow measurement technique and perfusion system described in this work are as yet specifically aimed at the development of (sub)normothermic preservation techniques in the experimental setting in a non-portable setup.

In conclusion, flow sensors based on the Coriolis technique measure flow rate more accurately without the need for recalibration regardless of preservation solution or temperature employed during machine perfusion. The presented preservation system proved to provide accurate flow and pressure management throughout temperature and preservation solution changes. The systems' stability was hereafter confirmed with the perfusion of NHBD kidneys, safeguarding extrapolation of our results to the setting of organ perfusion preservation. Moreover, the system could comply with preprogrammed flow- and pressurewaveforms, facilitating the reproduction of defined flow and pressure waveforms of experimental and clinical machine perfusion devices. This project therefore resulted in a system capable of looking into 
(a)

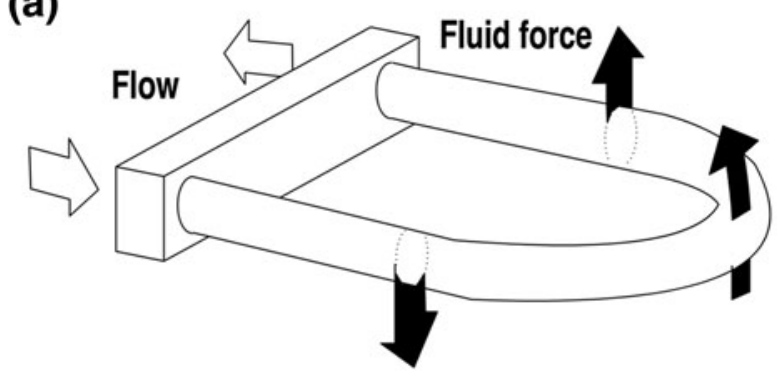

(b)

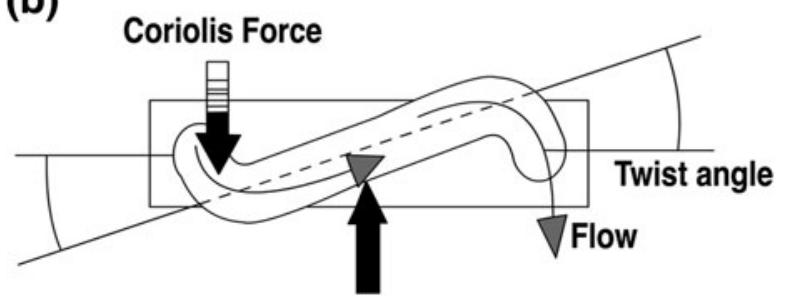

FIGURE 6. Principle of the Coriolis mass flow measurement, illustrating the forces and flow tube torsion when flow is present.

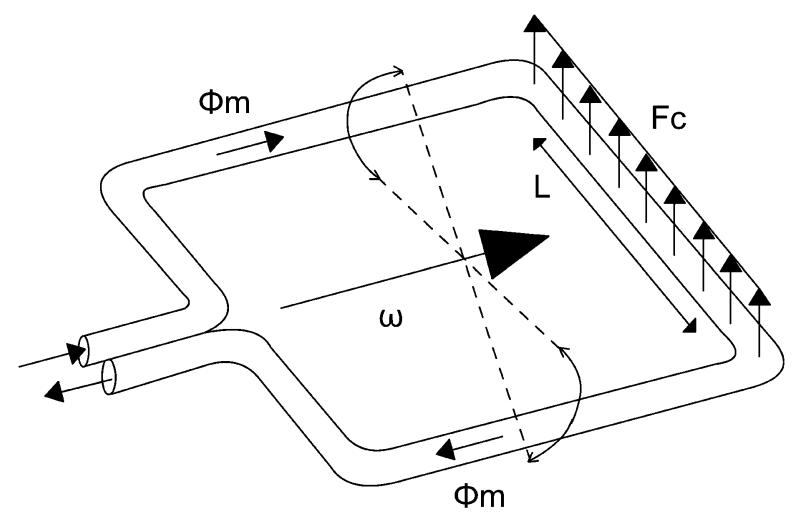

FIGURE 7. Coriolis flow sensor in which $\omega$ is the torsion mode actuation vector, $F_{\mathrm{c}}$ indicates the Coriolis force as a result of $\Phi_{m}$, being mass flow.

optimal machine perfusion settings in a way that flow and pressure waveforms might be pre-validated.

\section{ACKNOWLEDGMENTS}

We thank Marcel Katerberg (Bronkhorst BV) and Christiane Custers (Transonic) for their technical assistance during this study.

\section{CONFLICT OF INTEREST}

The author (IC Post) received technical support from Bronkhorst High-Tech, the manufacturer of the Coriolis-based flow sensor, but without benefits or restrictions in the publication of results.

\section{OPEN ACCESS}

This article is distributed under the terms of the Creative Commons Attribution License which permits any use, distribution, and reproduction in any medium, provided the original author(s) and the source are credited.

\section{REFERENCES}

${ }^{1}$ Anklin, M., W. Drahm, and A. Rieder. Coriolis mass flowmeters: overview of the current state of the art and latest research Flow. Meas. Instrum. 17:317-323, 2006.

${ }^{2}$ Baker, R. C. Flow Measurement Handbook: Industrial Designs, Operating Principles, Performance, and Applications. Cambridge: Cambridge University Press, 2000.

${ }^{3}$ Belzer, F. O., and J. H. Southard. The future of kidney preservation. Transplantation 30:161-165, 1980.

${ }^{4}$ Brook, N. R., A. J. Knight, and M. L. Nicholson. Intrarenal resistance reflects warm ischaemic damage, and is further increased by static cold storage: a model of nonheart-beating donor kidneys. Med. Sci. Monit. 9:BR271BR275, 2003.

${ }^{5}$ Cypel, M., J. C. Yeung, M. Liu, et al. Normothermic ex vivo lung perfusion in clinical lung transplantation. N. Engl. J. Med. 364:1431-1440, 2011.

${ }^{6} \mathrm{de}$ Vries, E. E., E. R. Hoogland, B. Winkens, et al. Renovascular resistance of machine-perfused DCD kidneys is associated with primary nonfunction. Am. J. Transplant. 11:2685-2691, 2011.

${ }^{7}$ Hosgood, S. A., and M. L. Nicholson. First in man renal transplantation after ex vivo normothermic perfusion. Transplantation 92:735-738, 2011.

${ }^{8}$ Hosgood, S. A., and M. L. Nicholson. Normothermic kidney preservation. Curr. Opin. Organ. Transplant. 16:169-173, 2011.

${ }^{9}$ Jochmans, I., C. Moers, J. M. Smits, et al. The prognostic value of renal resistance during hypothermic machine perfusion of deceased donor kidneys. Am. J. Transplant. 11:2214-2220, 2011.

${ }^{10}$ McLelland, S. J., and A. P. Nicholas. A new method for evaluating errors in high-frequency ADV measurements. Hydrol. Process. 14:351-366, 2000.

${ }^{11}$ Nieuwoudt, M., G. H. C. Engelbrecht, L. Sentle, et al. Non-toxicity of IV injected perfluorocarbon oxygen carrier in an animal model of liver regeneration following surgical injury. Artif. Cells Blood Substit. Immobil. Biotechnol. 37:117-124, 2009.

${ }^{12}$ Olschewski, P., P. Gass, V. Ariyakhagorn, et al. The influence of storage temperature during machine perfusion on preservation quality of marginal donor livers. Cryobiology 60:337-343, 2010.

${ }^{13}$ Oosterlee, A., and A. Rahmel. Annual Report 2010. Leiden: Eurotransplant International Foundation, 2011.

${ }^{14}$ Post, I. C., M. C. Dirkes, M. Heger, et al. Appraisal of the porcine kidney autotransplantation model. Front. Biosci. 4:1345-1357, 2012.

${ }^{15}$ Schreinemachers, M. C., B. M. Doorschodt, S. Florquin, et al. Pulsatile perfusion preservation of warm ischaemiadamaged experimental kidney grafts. Br. J. Surg. 97:349$358,2010$. 
${ }^{16}$ Shikanov, S., M. Wille, M. Large, et al. Microparticulate ice slurry for renal hypothermia: laparoscopic partial nephrectomy in a porcine model. Urology 76:1012-1016, 2010.

${ }^{17}$ Sung, R. S., L. L. Christensen, A. B. Leichtman, et al. Determinants of discard of expanded criteria donor kidneys: impact of biopsy and machine perfusion. Am. J. Transplant. 8:783-792, 2008.

${ }^{18}$ Thomas, L. H. The dependence of the viscosities of liquids on reduced temperature, and a relation between viscosity, density, and chemical constitution. J. Chem. Soc. 29:573579, 1946.

${ }^{19}$ Timsit, M. O., and S. G. Tullius. Hypothermic kidney preservation: a remembrance of the past in the future? Curr. Opin. Organ. Transplant. 16:162-168, 2011.
${ }^{20}$ Tolboom, H., M. L. Izamis, N. Sharma, et al. Subnormothermic machine perfusion at both $20^{\circ} \mathrm{C}$ and $30^{\circ} \mathrm{C}$ recovers ischemic rat livers for successful transplantation. J. Surg. Res. 175(1):149-156, 2011.

${ }^{21}$ Vogel, T., J. G. Brockmann, C. Coussios, and P. J. Friend. The role of normothermic extracorporeal perfusion in minimizing ischemia reperfusion injury. Transplant. Rev. 26:156-162, 2012.

${ }^{22}$ White, C. R., N. F. Phillips, and R. S. Seymour. The scaling and temperature dependence of vertebrate metabolism. Biol. Lett. 2:125-127, 2006.

${ }^{23}$ Znaien, J., Y. Hallez, F. Moisy, et al. Experimental and numerical investigations of flow structure and momentum transport in a turbulent buoyancy-driven flow inside a tilted tube. Phys. Fluids 21:115102, 2009. 